\title{
Improved Iterative Coordinated Beamforming Based on Singular Value Decomposition for Multiuser Mimo Systems With Limited Feedforward
}

\author{
L. Soriano-Equigua* ${ }^{* 1}$, J. Sánchez-García ${ }^{2}$, C.-B. Chae ${ }^{3}$, R. W. Heath Jr. ${ }^{4}$ \\ ${ }^{1,2}$ Centro de Investigación Científica y de Educación Superior de Ensenada (CICESE), \\ Carretera Ensenada-Tijuana No. 3918, Zona Playitas, \\ Ensenada, B. C. México. C.P. 22860. \\ *Isoriano@cicese.mx \\ ${ }^{3}$ School of Integrated Technology, College of Engineering, \\ Yonsei University, 162-1 Songdo-dong, Yeonsu-gu, \\ Incheon, 406-840, Korea \\ ${ }^{4}$ Wireless Networking and Communications Group (WNCG), \\ Department of Electrical and Computer Engineering, \\ The University of Texas at Austin, \\ 1 University Station C0803, Austin, TX, USA 78712.
}

\begin{abstract}
Coordinated beamforming based on singular value decomposition is an iterative method to jointly optimize the transmit beamformers and receive combiners, to achieve high levels of sum rates in the downlink of multiuser systems, by exploiting the multi-dimensional wireless channel created by multiple transmit and receive antennas. The optimization is done at the base station and the quantized beamformers are sent to the users through a low rate link. In this work, we propose to optimize this algorithm by reducing the number of iterations and improving its uncoded bit error rate performance. Simulation results show that our proposal achieves a better bit error rate with a lower number of iterations than the original algorithm.
\end{abstract}

Keywords: Coordinated beamforming, multiuser MIMO, iterative, convergence, bit error rate.

\section{RESUMEN}

El beamforming coordinado basado en descomposición de matrices en valores singulares, es un método iterativo que nos permite calcular conjuntamente los vectores de peso de las antenas transmisoras en la estación base y las antenas receptoras en los móviles, para alcanzar altos niveles de tasas de bits en el canal de bajada de sistemas MIMO multiusuario. La optimización se realiza en la estación base y los vectores de peso cuantizados del transmisor se envían a cada usuario a través de un enlace de baja velocidad. En este trabajo, nosotros proponemos optimizar este algoritmo para reducir el número de iteraciones necesarias para que el algoritmo converja y mejorar la tasa de bit errónea. Los resultados de las simulaciones realizadas muestran que nuestra propuesta alcanza una mejor tasa de bit errónea con un menor número de iteraciones.

\section{Introduction}

Multiple-input multiple-output (MIMO) communication is widely acknowledged as the key technology for achieving high data rates in wireless communication systems. MIMO systems can increase the channel capacity and link robustness of wireless communication by exploiting the multi-dimensional wireless channel created by multiple transmit and receive antennas [1]-[3]. Multiuser MIMO (MU-MIMO) systems combine the high capacity achievable with MIMO systems with the benefits of space division multiple access (SDMA). Such systems consider a base station that transmits to multiple mobile stations simultaneously over the same frequency band, with a substantially increase in the channel capacity compared to other multi-access schemes such as conventional TDMA [4].

Dirty paper coding (DPC) is the optimal strategy given by information theory for the MIMO broadcast channel, where it achieves the maximum sum rate, 
however it is difficult to implement due to its high complexity [5]. Several practical near-DPC techniques based on the concept of precoding have been proposed with different tradeoffs between complexity and performance. One of the simplest approaches for multiuser precoding is to premultiply the transmitted signal by a suitably normalized inverse of the multiuser channel matrix through zero forcing or minimum mean square error (MMSE) [6]. They have the advantage of being relatively easy to implement, but require one receive antenna per user. Other proposal is called block diagonalization, that enforces a zero interference property at each user but requires the number of receive antennas to be equal to the number of data streams for each user. Coordinated beamforming (CBF), a generalization of block diagonalization, provides high sum rates for downlink communication in the MU-MIMO channel and does not impose any restriction on the number of receive antennas subject to send one stream of data per user. The benefits of CBF are a result of jointly optimizing both transmit beamforming and receive combining vectors [7][12]. The basic concept of this kind of algorithms is to use a group of transmit beamforming vectors that ensure zero inter-user interference and a group of receive combining vectors that maximize the sum rate of the system.

One of the simplest approaches for CBF is the iterative algorithm based on singular value decomposition (CBF-SVD) proposed in [10]. This approach takes advantage of the full channel state information $(\mathrm{CSI})$ at the transmitter to maximize the sum rate.

Particularly in time division duplexing (TDD) systems, it is possible to obtain the full CSI by employing the reciprocity concept at the base station (BS), which means that the transmit channel is inferred based on an estimate of the received channel when the time between transmission and reception is smaller than the channel coherence time, at the cost of a careful system design and calibration. In this scenario, the BS gets all user's channel information and each user can only estimate its own channel information, this allows the optimization to be done at the BS with the challenge that each user needs to figure out which is the correct receive combining vector. To solve this, a limited feedforward technique was proposed in [10]. Throughout this paper, we assume that only a common pilot channel is available in the system. In this case, there is no way to estimate the receive combining vector without using a feedforward message. The Limited Feedforward Technique takes advantage of the overhead reduction achieved by using Grassmannian codebooks and quantizes the beamforming vector that each receiver uses to compute its combining vector; the quantization procedure aims to maximize the sum rate of the system. The quantized vectors are chosen from a codebook known both at the BS and the mobile station (MS), and the BS just sends the index of the corresponding codes through a feedforward link.

Several algorithms have been proposed for CBF with limited feedforward (see [10] and [11]); from the performance evaluation of these algorithms, it has been shown that the iterative algorithms achieve a better performance in terms of sum rate. However, due to their iterative nature, the complexity increases with the number of iterations. In this work, we propose to optimize the CBF-SVD algorithm by improving the BER performance and reducing the necessary number of iterations, while achieving the same sum rate. Furthermore, the simulation results showed that the convergence of the algorithm was improved.

Notation: We use uppercase and lowercase boldface letters to denote matrices and vectors, respectively. We denote the transpose, Hermitian, singular value decomposition, and 2-norm of $\mathbf{A}$ by $\mathbf{A}^{T}, \mathbf{A}^{H}$, $\operatorname{svd}(\mathbf{A})$, and $\|\mathbf{A}\|$, respectively. We denote the dot product between $\mathbf{a}$ and $\mathbf{b}$ by $\langle\mathbf{a}, \mathbf{b}\rangle$.

\section{System model and cbf-svd algorithm review}

In this section, we provide a short description of the multiuser MIMO system and give a review of the coordinated beamforming algorithm presented in [10].

\subsection{System model}

Consider the downlink transmission of a multiuser MIMO system with $N_{t}$ transmission antennas, $N_{r}$ receive antennas, and $\mathrm{K}$ users $\left(\mathrm{K} \leq \mathrm{N}_{\mathrm{t}}\right)$, as illustrated in Figure 1. Before transmission each complex symbol $x_{k}$ generated to the kth $(1 \leq k \leq K)$ user is multiplied by a beamforming vector $f_{k}$, then all the 


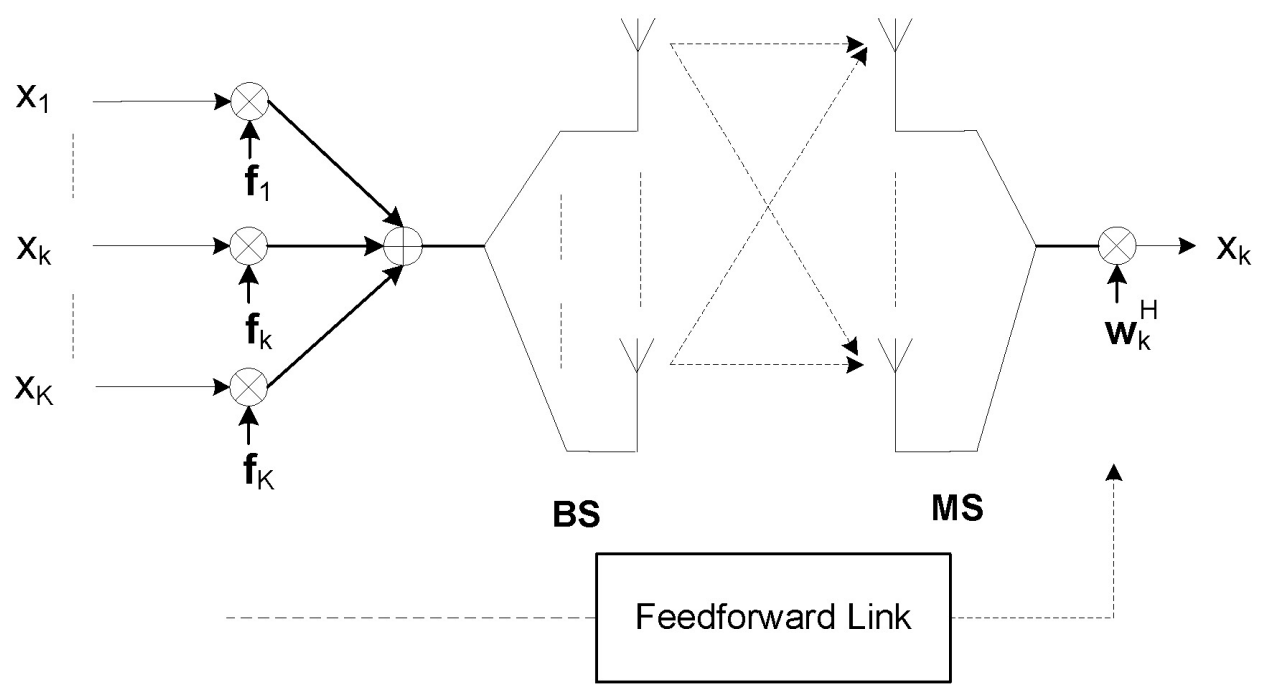

Figure 1. Coordinated beamforming system model with limited feedforward.

resulting vectors are added and each complex scalar is sent to the corresponding antenna. The base station launches the resulting signal into the propagation environment. The necessary information to calculate the combiners is sent to each user through a limited feedforward link, which is assumed with zero delay. The channel is assumed to be constant during the frame transmission time. We assume that the power is equally allocated across all users.

The signal $y_{k}$ received by the $k$ th user after applying the combining vector $\mathbf{w}_{k}$ is given by

$$
y_{k}=\mathbf{w}_{k}^{H} \mathbf{H}_{k} \mathbf{f}_{k} \tilde{x}_{k}+\mathbf{w}_{k}^{H} \mathbf{H}_{k} \sum_{\ell=1 \ell \neq k}^{K} \mathbf{f}_{\ell} \tilde{x}_{\ell}+\mathbf{w}_{k}^{H} \mathbf{v}_{k}
$$

where $\mathbf{v}_{k}$ is a vector of independent identically distributed (i.i.d.) complex zero-mean Gaussian noise with variance $\sigma^{2}, \mathbf{H}_{k}$ is the channel for the $k t h$ mobile station (MS) represented by a matrix of size $N_{r} \times N_{t} . \mathbf{f}_{\mathrm{k}}$ and $\mathbf{w}_{k}$ are the transmit beamforming vectors and receive combining vectors, respectively, calculated by using coordinated beamforming.
In Equation (1), the first term represents the effective channel gain for the $k$ th user, the second term shows the multi-user interference, and the last term illustrates the vector noise multiplied by $\mathbf{w}_{k}$. As $\mathbf{f}_{k}$ and $\mathbf{w}_{k}$ are unitary vectors, the noise is not amplified at the receiver when the combining vector is applied.

\subsection{Coordinated beamforming algorithm review}

The iterative algorithm to compute the beamformers and combiners, subject to sum rate maximization proposed in [10], is shown in Figure 2 and is summarized as follows:

1) Initialize the combining vectors of the users to unit vectors. A good initialization is by setting $\mathbf{w}_{k}$ to the left singular vector that corresponds to the maximum singular value of $\mathbf{H}_{k}$, that is

$$
\begin{aligned}
& {\left[\begin{array}{lll}
\mathbf{U} & \mathbf{D} & \mathbf{V}
\end{array}\right]=\operatorname{svd}\left(\mathbf{H}_{k}\right)} \\
& \mathbf{w}_{k}=\mathbf{U}[:, 1]
\end{aligned}
$$

where $\mathbf{U}[:, 1]$ represents the first column vector of $\mathbf{U}$. 
2) Form the effective channel matrix as

$$
\left.\tilde{\mathbf{H}}_{k}=\left[\begin{array}{llllll}
\left(\mathbf{w}_{1}^{H} \mathbf{H}_{1}\right)^{T} & \cdots & \left(\mathbf{w}_{k-1}^{H} \mathbf{H}_{k-1}\right)^{T} & \left(\mathbf{w}_{k+1}^{H} \mathbf{H}_{k+1}\right)^{T} & \cdots & \left(\mathbf{w}_{k}^{H} \mathbf{H}_{k}\right.
\end{array}\right)^{T}\right]^{T} .
$$

3) Calculate the beamforming vector $\mathbf{f}_{k}$ such that it cancels the interference between users. The interference cancelation is done by calculating a vector orthogonal to the rows of $\tilde{\mathbf{H}}_{k}$; this is realized by calculating the SVD of $\tilde{\mathbf{H}}_{k}$ and taking as beamformer the right singular vector that corresponds to the singular value zero (the size of $\tilde{\mathbf{H}}_{k}$ is $(K-1) \times N_{t}$, if we consider $K=N_{t}$ the existence of one zero singular value is guaranteed). Then,

$$
\begin{aligned}
& {\left[\begin{array}{lll}
\mathbf{U} & \mathbf{D} & \mathbf{V}
\end{array}\right]=\operatorname{svd}\left(\tilde{\mathbf{H}}_{k}\right)} \\
& \mathbf{f}_{k}=\mathbf{V}\left[:, N_{t}\right]
\end{aligned}
$$

where $\mathbf{V}\left[:, N_{t}\right]$ represents the last column vector of $\mathbf{V}$ (in SVD the right singular vectors are sorted such that the first column vector corresponds to the larger singular value and the last corresponds to the smaller singular value, in this case equal to zero).

4) Check the stopping criteria. The algorithm stops when the maximum allowed number of iterations is achieved or the difference between the previous and current beamformers is small enough.

$$
\left\|\mathbf{f}_{k, i}-\mathbf{f}_{k, i-1}\right\|<\varepsilon
$$

where $i$ and $i-1$ represent the actual and previous iteration, respectively.

5) Otherwise update the combining vectors using maximum ratio combining (MRC)

$$
\mathbf{w}_{k}=\mathbf{H}_{k} \mathbf{f}_{k} \text {, }
$$

and go to step 2 .

6) Quantize the beamformers. The beamformers are complex vectors that can take an infinite number of values; to send the index of the code that represents the current beamformer value, they need to be quantized. To find the quantized beamformers $\hat{\mathbf{f}}_{k}$, the transmitter selects the codebook index corresponding to the code that maximizes the signal-to-interference-plus-noise ratio $(S I N R)$

$$
\hat{\mathbf{f}}_{k}=\underset{\mathbf{c}_{i} \in \mathcal{C}}{\operatorname{argmax}} \operatorname{SINR} R_{k}\left(\mathbf{c}_{i}\right),
$$

where

$$
\begin{aligned}
& \operatorname{SINR}_{k}\left(\mathbf{c}_{i}\right)= \\
& \frac{\left|\mathbf{c}_{i}^{H} \mathbf{R}_{k} \mathbf{f}_{k}\right|^{2}}{\mathbf{c}_{i}^{H}\left(\sum_{l=1, I \neq k}^{K} \mathbf{R}_{k} \mathbf{f}_{l} \mathbf{f}_{l}^{H} \mathbf{R}_{k}\right) \mathbf{c}_{i}+\mathbf{c}_{i}^{H} \mathbf{R}_{k} \mathbf{c}_{i} \sigma^{2}},
\end{aligned}
$$

$\mathbf{R}_{k}=\mathbf{H}_{k}^{H} \mathbf{H}_{k}$ is the matched channel matrix and $\mathcal{C}$ is a codebook that was generated using techniques in [13].

7) Cancel residual interference. After quantization, the BS updates the combiners, and executes steps two and three again to mitigate residual interference due to the quantization operation; we denote the final beamformers as $\left\{\tilde{\mathbf{f}}_{k}\right\}_{k=1}^{K}$.

\section{Improved cbf-svd algorithm}

In the previous section, the CBF-SVD approach was reviewed, which is a transmitter based iterative technique for multiuser beamforming that improves the SINR in the system. After each iteration and quantization, the beamforming vectors are optimized in terms of sum rate. In this section, we show a method to improve the system BER performance by selecting the proper beamforming vectors. In this paper, the optimization objective is to minimize the BER, which is equivalent to minimize the phase noise derived from applying the resulting beamformers at the transmitter and the combiners at the receivers. 


\subsection{Analysis of the original algorithm}

Essentially, the algorithm described in Section 2 imposes zero interference iteration by iteration and evaluates the squared root of the error in the obtained beamformers to determine the convergence of the algorithm. The beamforming vectors are selected to cancel the interference after the combining vectors are calculated. To illustrate the effects of the calculation of the beamformers in this way, we analyze the case of $N_{t}=N_{r}=K=4$.

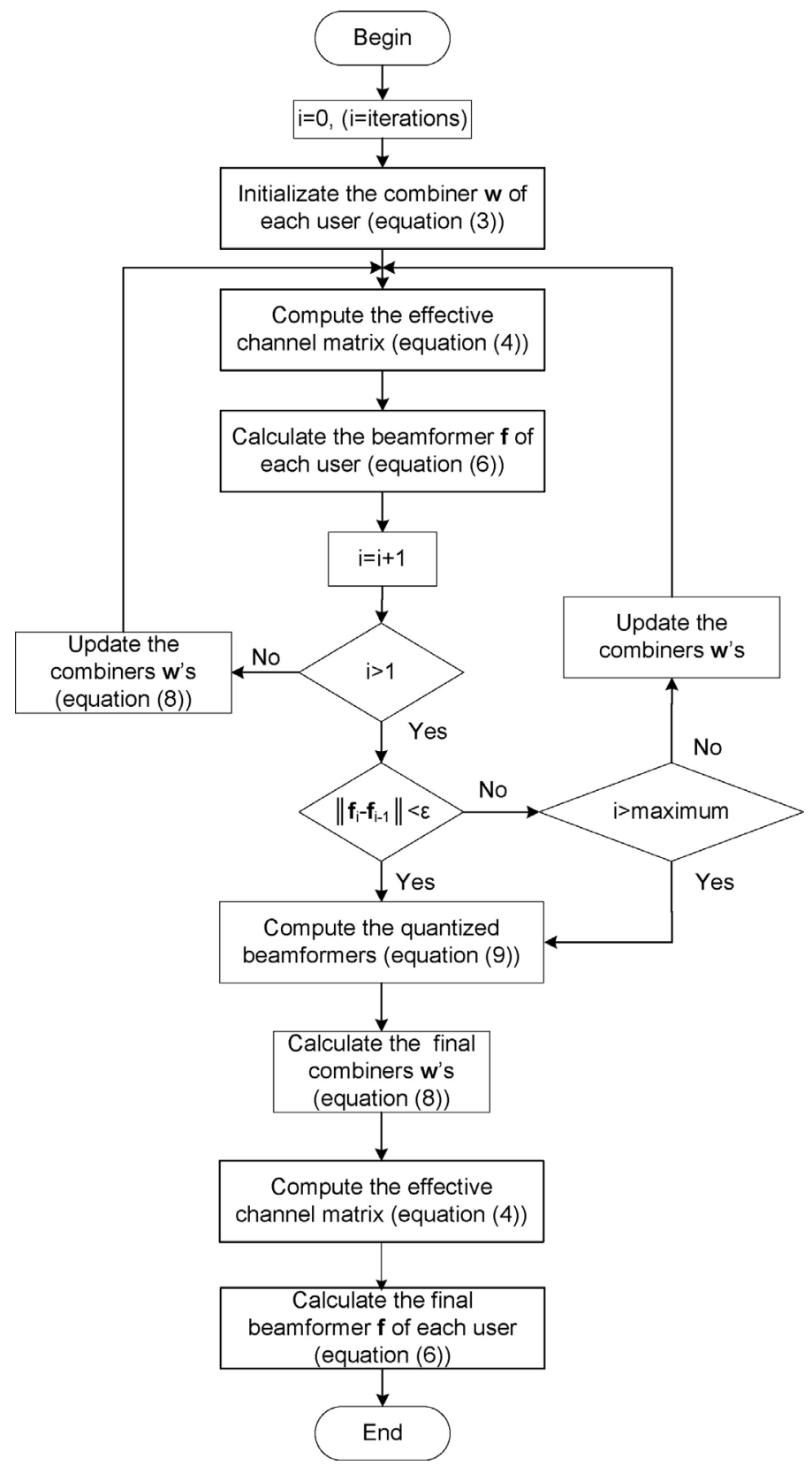

Figure 2. Flow diagram of the CBF-SVD algorithm. 
Consider the ith iteration, the matrix $\tilde{\mathbf{H}}_{k}$ (with $k=2$ ) is conformed as

$$
\tilde{\mathbf{H}}_{2}=\left[\begin{array}{cccc}
\tilde{h}_{1,1} & \tilde{h}_{1,2} & \tilde{h}_{1,3} & \tilde{h}_{1,4} \\
\tilde{h}_{3,1} & \tilde{h}_{3,2} & \tilde{h}_{3,3} & \tilde{h}_{3,4} \\
\tilde{h}_{4,1} & \tilde{h}_{4,2} & \tilde{h}_{4,3} & \tilde{h}_{4,4}
\end{array}\right]
$$

where $\left[\begin{array}{llll}\tilde{h}_{k, 1} & \tilde{h}_{k, 2} & \tilde{h}_{k, 3} & \tilde{h}_{k, 4}\end{array}\right]=\mathbf{w}_{k}^{H} \mathbf{H}_{k}$.

To cancel interference, the beamforming vector that belongs to the second user satisfies

$$
\left[\begin{array}{cccc}
\tilde{h}_{1,1} & \tilde{h}_{1,2} & \tilde{h}_{1,3} & \tilde{h}_{1,4} \\
\tilde{h}_{3,1} & \tilde{h}_{3,2} & \tilde{h}_{3,3} & \tilde{h}_{3,4} \\
\tilde{h}_{4,1} & \tilde{h}_{4,2} & \tilde{h}_{4,3} & \tilde{h}_{4,4}
\end{array}\right]\left[\begin{array}{l}
f_{2,1} \\
f_{2,2} \\
f_{2,3} \\
f_{2,4}
\end{array}\right]=\left[\begin{array}{l}
0 \\
0 \\
0
\end{array}\right]
$$

this is achieved by setting the beamforming vector as the right singular vector that corresponds to the singular value zero. As a consequence, Equation (1) becomes

$$
y_{k}=\mathbf{w}_{k}^{H} \mathbf{H}_{k} \mathbf{f}_{k} \tilde{x}_{k}+\mathbf{w}_{k}^{H} \mathbf{v}_{k} .
$$

Without interference the SINR is reduced to

$$
\operatorname{SINR} R_{k}=\frac{\left|\mathbf{w}_{k}^{H} \mathbf{H}_{k} \mathbf{f}_{k}\right|^{2}}{\sigma_{k}^{2}}
$$

where $\left|\mathbf{w}_{k}^{H} \mathbf{H}_{k} \mathbf{f}_{k}\right|^{2}$ is the channel effective gain. It is important to mention that to correctly decoding the transmitted symbol $\boldsymbol{x}_{k}$, the product $\mathbf{w}_{k}^{H} \mathbf{H}_{k} \mathbf{f}_{k}$ must be real and positive, otherwise the received symbol $y_{k}$ will present phase noise and the BER performance will be degraded.

The algorithm explained in Section 2 ensures the interference cancelation but $\mathbf{w}_{k}^{H} \mathbf{H}_{k} \mathbf{f}_{k}$ is not guaranteed to result in a real and positive scalar, as a result $\mathbf{f}_{k}$ is not optimized in terms of the BER performance. Computing $\mathbf{f}_{k}$ as in [10] also impacts the convergence because the algorithm does not compare optimal beamformers to determine when the iterative process must stop.

3.2 Nonuniqueness of the beamformers to cancel multiuser interference

The beamformers that cancel the interference in the iterative process of CBF-SVD and maximize the SINR are not unique, quite the opposite there is a family of vectors $\mathbf{f}_{k} e^{-j \theta_{k}} \quad\left(0 \leq \theta_{k} \leq 2 \pi\right)$, for each beamformer as is shown next.

Lema 1: If $\mathbf{f}_{k}$ is orthogonal to $\left\{\mathbf{w}_{l}^{H} \mathbf{H}_{l}\right\}_{l=1, l \neq k}^{K}$, then $\mathbf{f}_{k} e^{-j \theta_{k}}$ is also orthogonal to it for all $\theta_{k}$.

Proof: The proof of this lemma is straightforward by applying the properties of the dot product of vectors and the norm properties [14] to the complex angle between two vectors. Consider $l \neq k$ and $\mathbf{w}_{l}^{H} \mathbf{H}_{l} \mathbf{f}_{k}=0$ then from the equation for the complex angle between two vectors [15] we have

$$
\begin{aligned}
& \frac{\left\langle\mathbf{w}_{l}^{H} \mathbf{H}_{l}, \mathbf{f}_{k} e^{-j \theta_{k}}\right\rangle}{\left\|\mathbf{w}_{l}^{H} \mathbf{H}_{l}\right\|\left\|\mathbf{f}_{k} e^{-j \theta_{k}}\right\|}=0 \\
& \frac{e^{-j \theta_{k}}\left\langle\mathbf{w}_{l}^{H} \mathbf{H}_{l}, \mathbf{f}_{k}\right\rangle}{\left\|\mathbf{w}_{l}^{H} \mathbf{H}_{l}\right\|\left\|\mathbf{f}_{k}\right\|\left|e^{-j \theta_{k}}\right|}=0,
\end{aligned}
$$

since $\left|e^{-j \theta_{k}}\right|=1$ and passing $e^{-j \theta_{k}}$ to the right side the expression is reduced to

$$
\frac{\left\langle\mathbf{w}_{l}^{H} \mathbf{H}_{l}, \mathbf{f}_{k}\right\rangle}{\left\|\mathbf{w}_{l}^{H} \mathbf{H}_{l}\right\|\left\|\mathbf{f}_{k}\right\|}=\left(e^{-j \theta_{k}}\right)(0)=0
$$

From (13), we can see that the orthogonality is not affected by substituting $\mathbf{f}_{k}$ by $\mathbf{f}_{k} e^{-j \theta_{k}}$, thus if $\mathbf{f}_{k}$ is orthogonal to $\left\{\left(\mathbf{w}_{l}^{H} \mathbf{H}_{l}\right)^{T}\right\}_{l=1, l \neq k}^{K}, \quad \mathbf{f}_{k} e^{-j \theta_{k}}$ is orthogonal to $\left\{\left(\mathbf{w}_{l}^{H} \mathbf{H}_{l}\right)^{T}\right\}_{l=1, l \neq k}^{K}$.

Lema 2: The SINR achieved by using $\mathbf{f}_{k}$ or $\mathbf{f}_{k} e^{-j \theta_{k}}$ is the same. 
Proof: Since $e^{-j \theta_{k}}$ is a complex number with unitary absolute value, we have

$$
\begin{aligned}
\operatorname{SINR}_{k} & =\frac{\left|\mathbf{w}_{k}^{H} \mathbf{H}_{k} \mathbf{f}_{k} e^{-j \theta}\right|^{2}}{\sigma_{k}^{2}} \\
& =\frac{\left|\mathbf{w}_{k}^{H} \mathbf{H}_{k} \mathbf{f}_{k}\right|^{2}\left|e^{-j \theta}\right|^{2}}{\sigma_{k}^{2}} \\
& =\frac{\left|\mathbf{w}_{k}^{H} \mathbf{H}_{k} \mathbf{f}_{k}\right|^{2}}{\sigma_{k}^{2}}
\end{aligned}
$$

3.3 Proposed improvements to the cbf-svd algorithm

Given that the product $\mathbf{w}_{k}^{H} \mathbf{H}_{k} \mathbf{f}_{k}$ determines the BER performance, the objective of BER optimization is to choose the optimum beamformer $\mathbf{f}_{k} e^{-j \theta_{k}}$ that ensures that the product $\mathbf{w}_{k}^{H} \mathbf{H}_{k} \mathbf{f}_{k} e^{-j \theta}$ be real and positive.

\section{Problem statement:}

Find a phase $\theta_{k} \in\{0, \cdots, 2 \pi\}$ that minimize the absolute value of the complex angle between the vectors $\mathbf{w}_{k}^{H} \mathbf{H}_{k}$ and $\mathbf{f}_{k}$

$$
\theta_{k}=\underset{\theta}{\operatorname{argmin}} \mid \cos ^{-1}\left(\frac{\left\langle\mathbf{w}_{k}^{H} \mathbf{H}_{k}, \mathbf{f}_{k} e^{-j \theta_{k}}\right\rangle}{\left\|\mathbf{w}_{k}^{H} \mathbf{H}_{k}\right\|\left\|\mathbf{f}_{k} e^{-j \theta_{k}}\right\|} \mid\right.
$$

The solution to (14) is a kind of equalization and the angle $\theta_{k}$ is uniquely determined by

$\theta_{k}=\left\{\begin{array}{cc}\arctan \left(\operatorname{Im}\left(g_{k}\right) / \operatorname{Re}\left(g_{k}\right)\right) & \text { if } \operatorname{Re}\left(g_{k}\right)>0 \\ \arctan \left(\operatorname{Im}\left(g_{k}\right) / \operatorname{Re}\left(g_{k}\right)\right)+\pi & \text { if } \operatorname{Re}\left(g_{k}\right)<0 \\ 0 & \text { otherwise }\end{array}\right.$

where $g_{k}=\mathbf{w}_{k}^{H} \mathbf{H}_{k} \mathbf{f}_{k}$.

By considering the optimum $\mathbf{f}_{k} e^{-j \theta_{k}}$, we propose to improve the CBF-SVD algorithm as follows.

\section{At the Base Station}

1) Initialize the combining vectors for each user and obtain the beamformers as is done in the steps 1-3 of the CBF-SVD algorithm described in Section 2.

2) Optimize the beamformers by setting $\mathbf{f}_{k}=\mathbf{f}_{k} e^{-j \theta_{k}}$, where the $\theta_{k}$ parameter is calculated from (14).

3) Execute the steps 4-5 of the CBF-SVD algorithm described in Section 2 to determine if the algorithm stops or continues the iterative process.

4) Execute the steps 6-7 of the CBF-SVD algorithm and optimize the final beamformers $\left\{\tilde{\mathbf{f}}_{k}\right\}_{k=1}^{K}$ by substituting each one as $\tilde{\mathbf{f}}_{k} e^{-j \tilde{\theta}_{k}}$, where $\tilde{\theta}_{k}$ is calculated by replacing $\mathbf{w}_{k}$ with $\mathbf{H}_{k} \mathbf{c}_{i}$ and $\mathbf{f}_{k}$ with $\tilde{\mathbf{f}}_{k}$ in (14).

5) Send the codebook indices that correspond to the codes obtained in the quantization procedure to the mobile stations through the limited feedforward link.

\section{At the Mobile Station}

1) Calculate the combining vector applying

$$
\mathbf{w}_{k}=\frac{\mathbf{H}_{k} \mathbf{c}_{k}}{\left\|\mathbf{H}_{k} \mathbf{c}_{k}\right\|}
$$

2) Apply the combining vector to the received data to recover the transmitted data.

Our improved method achieves the same sum rate than CBF-SVD because the same SINR is achieved. The complexity increases by the included optimization, however, the necessary number of iterations is lower, and our proposal is optimized also in terms of BER performance.

\section{Simulation results}

Monte Carlo simulation results are presented in this section to demonstrate the performance of the improved CBF-SVD algorithm. The first subsection demonstrates its performance improvements in terms of average BER, in i.i.d. complex Gaussian channel 
and with perfect channel knowledge. The second subsection shows the performance of the iterative process in terms of average necessary number of iterations to converge. In all simulations we assume that i) the channels between different transmit and receive antenna pairs are independent, ii) the BS obtains a perfect channel estimate, iii) each user correctly estimates its own channel, and iv) all users share the same codebook.

\subsection{Bit error rate performance}

Figure 3 shows a comparison of the BER performance of a multiuser system where the BS and each user have four antennas. We consider that the number of users is equal to the number of antennas at the BS and we use QPSK modulation to compute the BER performance. Six cases are studied: a) unquantized original CBF-SVD (Obtained by executing steps 1-5 of the algorithm described in Section 2), b) unquantized improved CBF-SVD (Calculated by executing steps 1-3), c) quantized original CBF-SVD, d) quantized improved CBF-SVD, e) greedy-based quantization [11], f) iteration-based independent quantization (IBIQ) [11]. It is observed that our proposal has better BER for both quantized and non-quantized versions of CBF-SVD. The phase error in the original CBF-SVD algorithm does not permit to achieve good BER performance and to correct the resulting erroneous bits is almost impossible, even with error correction coding. Both quantized CBFSVD and improved quantized CBF-SVD show degradation in BER performance in comparison with its non-quantized versions, due to the quantization accuracy. As can be observed from Figure 3, the BER achieved by quantized improved CBF-SVD amply outperforms greedy-based quantization, while the BER performance is very close to that of IBIC. A loss in BER is observed when using four-bit codes instead of six-bit codes, as expected.

Figure 4 shows the BER performance for the case of a six-bit codebook, for the cases when $N_{t}=N_{r}=K$ and $K=2,3,4$. It can be seen that the average achieved BER performs better as the number of antennas and users increases, this is mainly due to the increase in the channel capacity. The worst performance is achieved by greedy-based quantization which shows no improvement as the number of antennas is increased, for SNR values greater than $16 \mathrm{dBs}$. As in Figure 3 , there is a marginal gap between improved CBF-SVD and IBIQ.

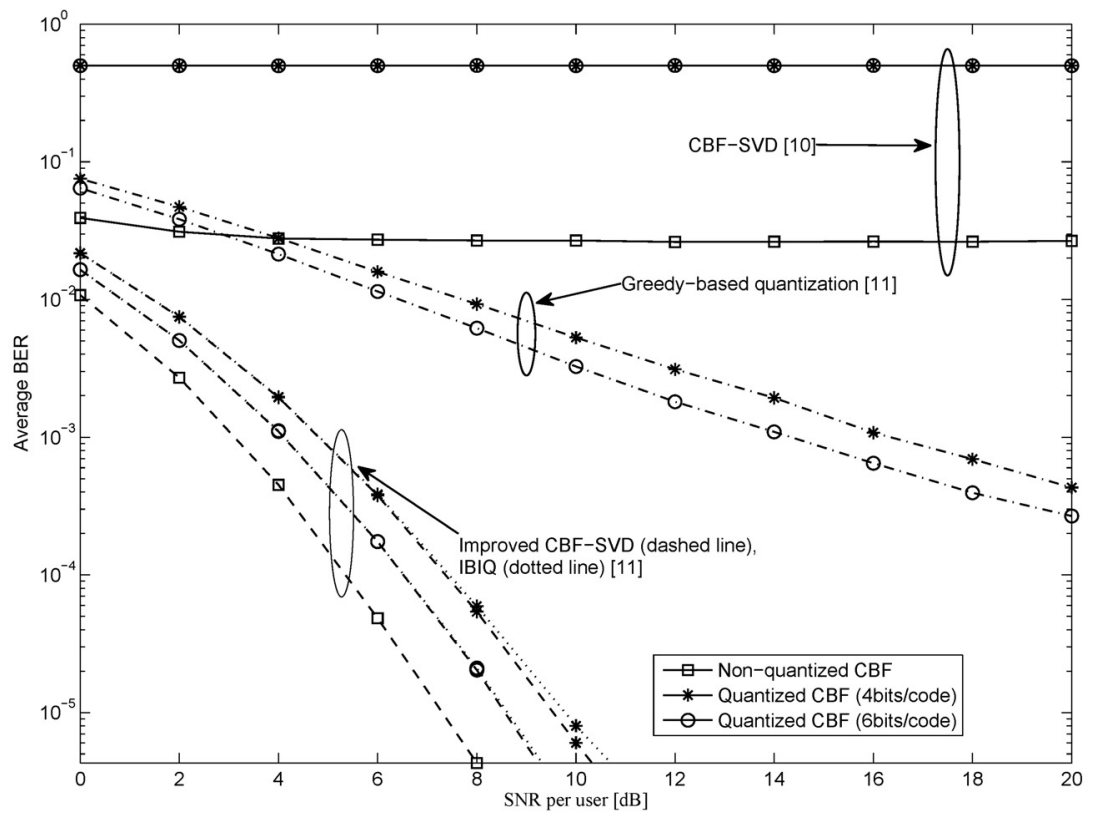

Figure 3. SNR vs BER when $N_{r}=N_{t}=K=4$. 


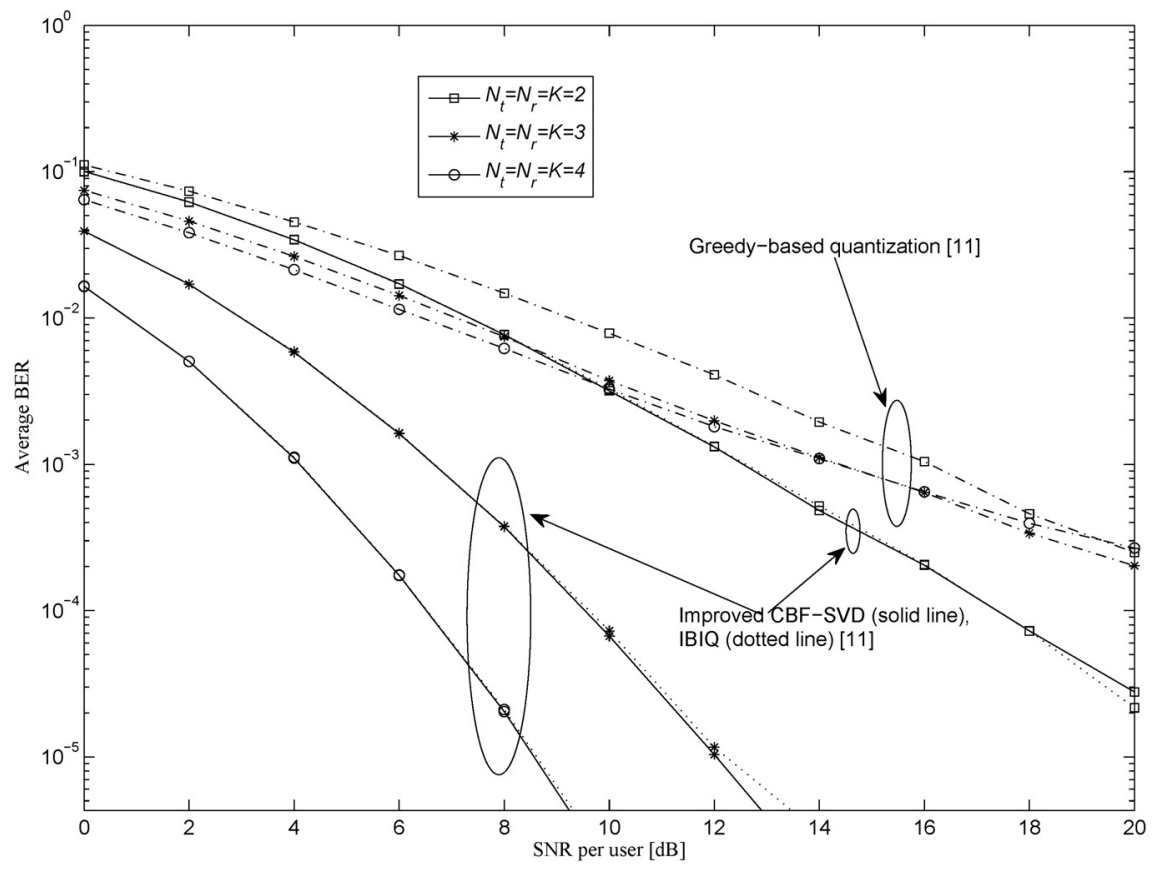

Figure 4. BER performance of improved CBF-SVD when $N_{r}=N_{t}=K$.

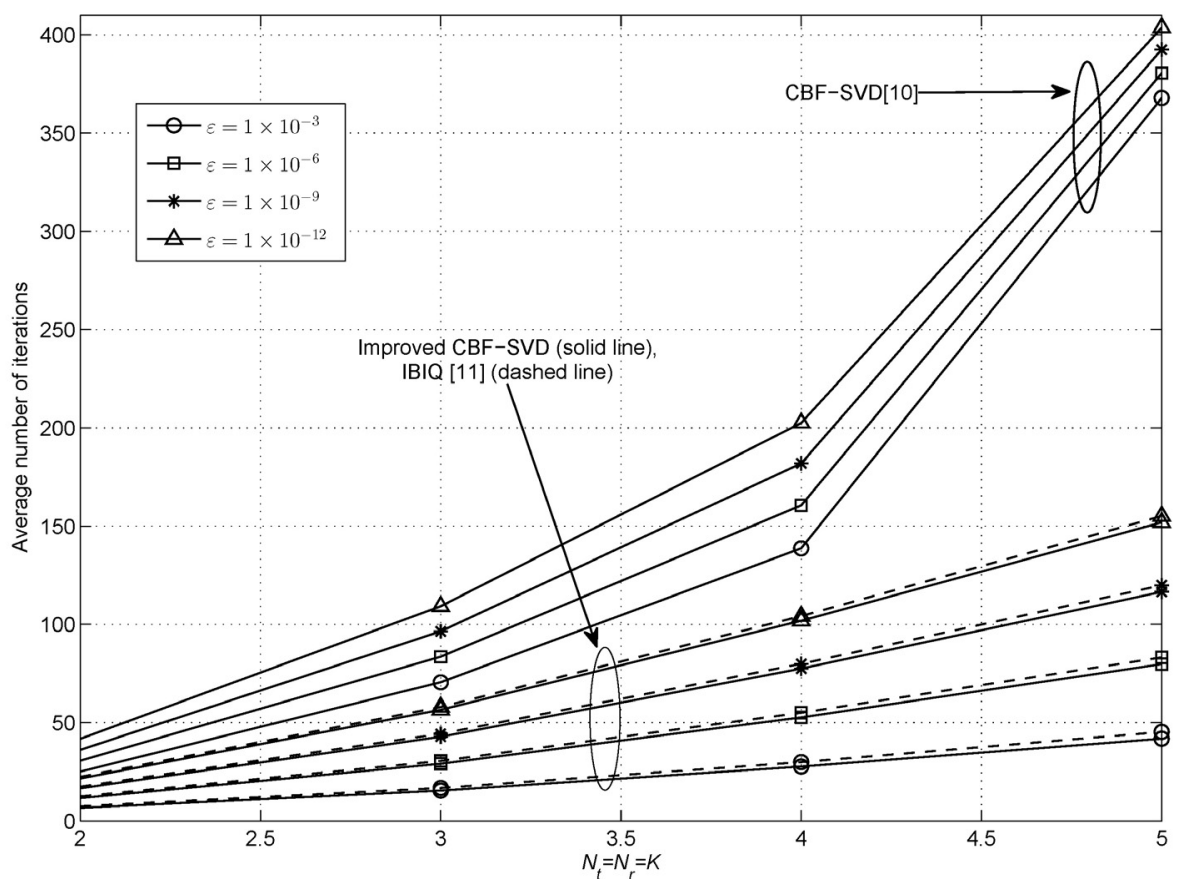

Figure 5. Convergence of improved CBF-SVD algorithm. 


\subsection{Convergence of the algorithm}

The convergence of both algorithms CBF-SVD and improved CBF-SVD is an important issue that can reduce the time of processing at the $B S$. This subsection provides numerical evaluation on the performance of improved CBF-SVD. Figure 5 shows average iterations versus number of users, we consider $N_{t}=N_{r}=K$ where $K=2,3,4,5$. We examine the performance under different values for the stop criteria $\varepsilon$ with 500 as the maximum number of possible iterations. In Figure 5, we can see from the curves that our proposal achieves the convergence faster than the original CBF-SVD for all values of $\varepsilon$. For the case $N_{t}=N_{r}=K=5$, our method converges with an average reduction of $62 \%$ in the iterations; for the other cases, it converges with almost $50 \%$ less than the average iterations computed by original CBF-SVD. Improved CBF-SVD slightly outperforms IBIQ for all considered cases.

\section{Conclusion and future work}

The proposed optimization to the CBF-SVD algorithm offers a better performance in terms of bit error rate with a lower number of iterations. The improvement in the number of iterations is in the order of $50 \%$ for $N_{t}=N_{r}=K=4$. We observed that for $10 \mathrm{~dB}$ of $S N R$ in $N_{t}=N_{r}=K=4$ configuration, improved CBF-SVD achieves an average BER up to $6 \times 10^{-6}$ (with six-bit codebook) while CBF-SVD achieves an average BER of 0.5 for all SNR values. Improved CBF-SVD achieves a better BER than greedy-based quantization at the cost of more complexity. Improved CBF-SVD slightly outperforms IBIQ in terms of convergence and both have a similar BER performance. In future work, we will evaluate the effect of user selection in the algorithm's convergence and the sum rate of the system.

\section{References}

[1] Telatar I. E., Capacity of multi-antenna Gaussian channels, European Transactions on Telecommunications, Nov. 1999, vol. 10, no. 6 , pp. 585-595.

[2] Foschini G. J. and Gans M. J., On limits of wireless communications in a fading environment when using multiple antennas, Wireless Networks Communications, Mar. 1998, vol. 6 no. 3, pp 311-335.

[3] Gesbert D., Shafi M., Shiu D.-S., Smith P. J., and Naguib A., From theory to practice: an overview of MIMO space-time coded wireless sytems, IEEE Journal on Selected Areas in Communications, Apr. 2003, vol. 21, no. 3, pp. 281-302.

[4] Spencer Q. H., Peel C. B., Swindlehurst A. L., and Haardt M., An introduction to the multi-user MIMO downlink, IEEE Communications Magazine, Oct. 2004, vol. 42 , no. 10 , pp. 60-67.

[5] Zhang C., Xu W., Chen M. Hybrid zero-forcing beamforming/orthogonal beamforming with user selection for MIMO broadcast channels, IEEE Communications Letters, Jan. 2009, vol. 13, no. 1, pp. 10-12.

[6] Peel C. B., Hochwald B. M., Swindlehurst A. L., A vector-perturbation technique for near-capacity multiantenna multiuser communication-part I: Channel inversion and regularization, IEEE Transactions on Communications, Jan. 2005, vol. 53, no. 1, pp. 195-202.

[7] Farhang-Boroujeny B., Spencer Q., and Swindlehurst A. L., Layering techniques for space-time communications in multi-user networks, in Proc. of IEEE Vehicular Technology Conference, Oct. 2003, vol. 2, pp. 1339-1342.

[8] Choi L. and Murch R.D., A transmit preprocessing technique for multiuser MIMO systems using a decomposition approach, IEEE Transactions on Wireless Communications, vol. 3, no. 1, Jan. 2004, pp. 20-24.

[9] Pan Z., Wong K.-K., and Ng T.-S., Generalized multiuser orthogonal space-division multiplexing, IEEE Transactions on Wireless Communications, vol. 3, Nov. 2004, pp. 1969-1973.

[10] Chae C.-B., Mazzarese D., and Heath R. W. Jr., Coordinated beamforming for multiuser mimo systems with limited feedforward. Proc. of IEEE Asilomar Conference on Signals, Systems, and Computers, 2006, pages 1511-1515, Pacific Grove, CA, Oct. 
[11] Chae C.-B., Mazzarese D., Inoue T., and Heath R. W. Jr., Coordinated beamforming for the multiuser MIMO broadcast channel with limited feedforward. IEEE Transactions on Signal Processing, Dec. 2008, vol. 56, no. 12 , pp. 6044-6056.

[12] Kim E.Y., Chun J. Coordinated beamforming with reduced overhead for the downlink of multiuser MIMO systems. IEEE Communication Letters, Nov. 2008, vol. 2, no. 11 , pp. $810-812$.

[13] Love D. J., Heath R. W. Jr., and Strohmer T., Grassmannian beamforming for multiple-input multipleoutput wireless systems, IEEE Transactions on Information Theory, vol. 49, Oct. 2003, pp. 2735-2747.

[14] Horn R. A. and Johnson C. R., Matrix analysis, 1st Ed., Cambridge university press, 1985, pp. 259-262.

[15] Scharnhorst K., Angles in Complex Vector Spaces, Acta Applicandae Mathematicae, Vol. 69, No. 1, October, 2001, pp. 95-103.

\section{Acknowledgements}

The work of C.-B. Chae was in part supported by the Ministry of Knowledge Economy under the "IT Consilience Creative Program" (NIPA-2010-C15151001-0001). 


\section{Authors' Biographies}

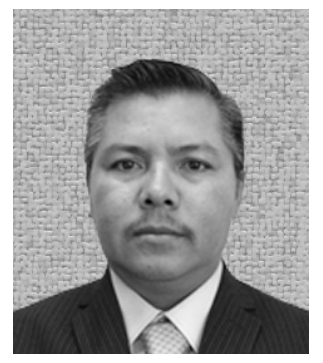

\section{LeoneI SORIANO-EQUIGUA}

Leonel Soriano-Equigua received the B.S. degree in communications and electronics engineering from Universidad de Colima in 1997, Colima, Mexico, and his M.Sc. degree in electronics and telecommunications from CICESE Research Center in 2000, in Ensenada, Baja California, Mexico. He is pursuing a Ph.D. degree at the same center. Leonel was an exchange visitor in the ECE department at The University of Texas at Austin in 2007. Since 2000, he has been an assistant professor in the Faculty of Mechanical and Electric Engineering at Universidad de Colima. His area of interest is MIMO communications.

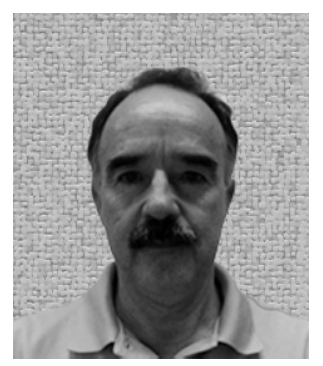

\section{Jaime SÁNCHEZ-GARCIIA}

Jaime Sanchez-García received an engineering degree in communications and electronics from Instituto Politécnico Nacional, IPN, Mexico City, Mexico, 1976; an M.Sc. degree in electronics and telecommunications from CICESE, Ensenada, Baja California, Mexico, 1979; and a D.Sc. degree in electrical engineering, with a major in communications, from The George Washington University, 2001. Since 1979, he has held a research and faculty position in the Electronics and Telecommunications Department at CICESE, Ensenada B.C., Mexico. Dr. Sánchez was a visiting scholar at University of Arizona, Tucson (ECE) in 1997, and at The University of Texas at Austin (ECE) in 2008. He has published several papers in IEEE Journals and International Conferences. His current research interests include wireless networks, software defined radio, channel modeling, multicarrier modulation (OFDM), and MIMO-OFDM. He is an IEEE member.

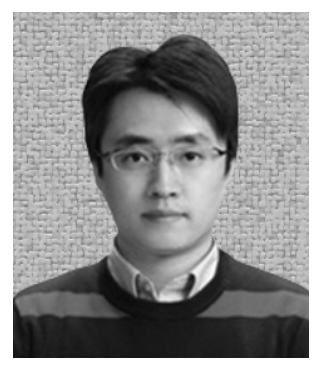

\section{Chan BYOUNG-CHAE}

Chan-Byoung Chae is an assistant professor at the School of Integrated Technology, College of Engineering, Yonsei University, Korea. He was a member of the technical staff (research scientist) at Bell Laboratories, AlcatelLucent, Murray Hill, NJ, USA, from June 2009 to February 2011. Before joining Bell Laboratories, he was with the School of Engineering and Applied Sciences at Harvard University, Cambridge, MA, USA, as a post-doctoral research fellow. He received the $\mathrm{Ph}$. D. degree in electrical and computer engineering from The University of Texas (UT), Austin, TX, USA in 2008, where he was a member of the Wireless Networking and Communications Group (WNCG). Prior to joining UT, he was a research engineer at the Telecommunications R\&D Center, Samsung Electronics, Suwon, Korea, from 2001 to 2005. He was a visiting scholar in the WING Laboratory, Aalborg University, Denmark in 2004 and at University of Minnesota, MN, USA in August 2007. While working at Samsung, he participated in the IEEE 802.16e 
standardization, where he made several contributions and filed a number of related patents from 2004 to 2005. His current research interests include capacity analysis and interference management in energy-efficient wireless mobile networks and all aspects of MIMO communications. He has served as an editor for the IEEE Trans. on Smart Grid.

Dr. Chae was the recipient of the IEEE Dan. E. Noble Fellowship Award in 2008, the Gold Prize (1st) in the 14th Humantech Thesis Contest, and the KSEA-KUSCO scholarship in 2007. He also received the Korea Government Fellowship (KOSEF) during his Ph. D. studies.

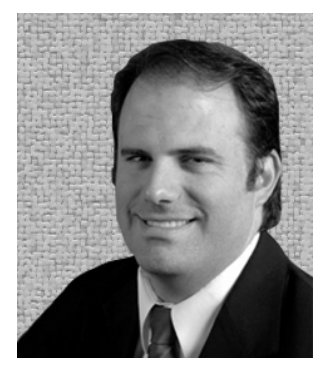

\section{Robert W. HEATH JR.}

Robert W. Heath Jr. received the Ph.D. degree from Stanford University, Stanford, CA, in 2002, in electrical engineering. He is with the Department of Electrical and Computer Engineering at The University of Texas at Austin where he is an associate professor. He is also president and CEO of MIMO Wireless Inc. and VP of Innovation at Kuma Signals LLC. Dr. Heath has been an editor for the IEEE Transactions on Communication, an associate editor for the IEEE Transactions on Vehicular Technology, lead guest editor for an IEEE Journal on Selected Areas in Communications special issue on limited feedback communication, and lead guest editor for an IEEE Journal on Selected Topics in Signal Processing special issue on heterogenous networks. He was a co-author of best student paper awards at IEEE VTC 2006 Spring, WPMC 2006, IEEE GLOBECOM 2006, IEEE VTC 2007 Spring, and IEEE RWS 2009, as well as co-recipient of the Grand Prize in the 2008 WinTech WinCool Demo Contest. He is the recipient of the David and Doris Lybarger Endowed Faculty Fellowship in Engineering, a licensed amateur radio operator, a registered professional engineer in Texas and a Fellow of the IEEE. 